\title{
Compromiso y educación. Redefinición del aula como contexto de aprendizaje de Daiana Y. Rigo (2020)*
}

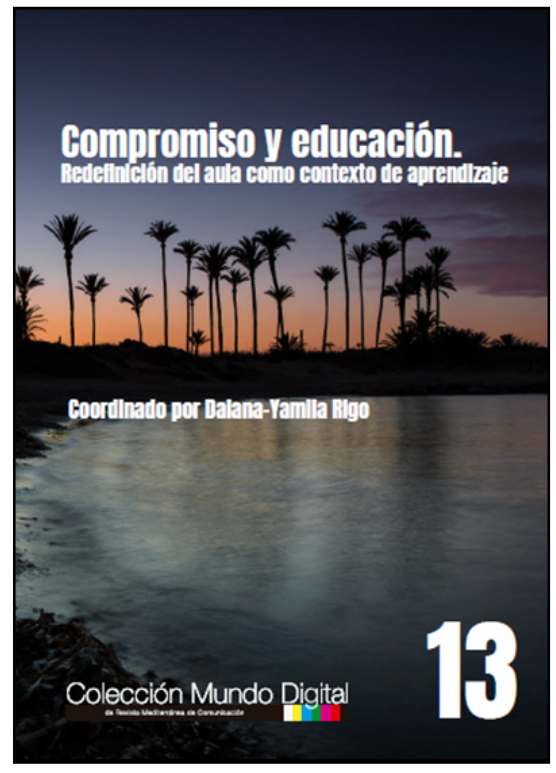

Cubierta del libro: Compromiso y educación. Redefinición del aula como contexto de aprendizaje

Fuente: Rigo (2020).

Daiana Schlegel

Universidad Nacional de Río Cuarto, Río Cuarto, Argentina daiana-schlegel@hotmail.com https://orcid.org/0000-0003-0583-5341

Cómo citar: Shlegel, D. (2021). Reseña del libro Compromiso y educación. Redefinición del aula como contexto de aprendizaje de Daiana-Y. (2020). Ciencias Sociales y Educación, 10(19), 397-403. https://doi.org/10.22395/ csye.v10n19a15

Recibido: 5 de octubre de 2020 .

Aprobado: 6 de octubre de 2020 . 
Cuando el contexto educativo se entiende como una red y las acciones se estudian como acontecimientos particulares, los vínculos entre la persona, las demandas de la tarea, los propósitos de la acción y las herramientas materiales o simbólicas disponibles pasan a ser objeto de mayor interés (Rinaudo, 2014). Entre las variables contextuales con reconocida incidencia, podemos mencionar la calidad de las tareas académicas, los procesos de feedback desplegados en clases y los modos de evaluación implementados. El compromiso consiste en esa energía que desata deseos de saber y mayor predisposición al esfuerzo que requiere el aprender.

La presente obra se enmarca en el campo de la psicología educacional que, fundamentada en la investigación científica, realiza aportes a la definición del compromiso académico. Así, desde enfoques o perspectivas socioculturales, se entiende al aprendizaje como un proceso eminentemente social que tiene lugar en las interacciones entre aprendices y las propiedades de ambientes específicos. En este marco, el libro comprende trabajos de reflexión teórica y de experiencias de investigación que se caracterizan por su rigor conceptual y metodológico.

La obra está compuesta por dos partes o secciones: una, predominantemente conceptual, denominada Propuestas diversas sobre compromiso; y la otra, principalmente empírica, titulada Investigar el compromiso como apuesta a la trasformación educativa. El prólogo es realizado por la docente Norma Susana Pereyra. El eje transversal del libro es el compromiso en el contexto educativo. En este sentido, se amplían las visiones al respecto y se brindan herramientas para promover el compromiso en contextos de enseñanza y de aprendizaje.

En la primera sección se estudia la conceptualización del compromiso desde diferentes perspectivas y, específicamente, se compone de tres capítulos. En el primer capítulo, denominado Compromiso. Como estilo de hacer de las maestras, Danilo Donolo presenta, en diferentes apartados, el hacer docente y las diversas experiencias que tienen como denominador común el compromiso. El trabajo no muestra una estructura tradicional académica, sino que va ilustrando en cada apartado las relaciones con el compromiso de diversas personas que se desempeñan en diferentes áreas. Por ejemplo, el autor resalta la impronta de su propia historia de formación en el compromiso asumido con la tarea de enseñar; presenta la historia de Eva Salanueva (maestra argentina recibida en el año 1920) y replantea la concepción del compromiso en aquellas épocas; comparte algunos comentarios sobre el trabajo de investigación de la coordinadora del libro, Daiana Rigo; y hace referencia a documentos académicos actuales en los que se esbozan conceptualizaciones sobre del compromiso, como los handbooks. También destina gran parte del texto a la visión que se tiene del compromiso 
desde las políticas públicas. En este sentido, reflexiona sobre problemáticas vigentes, tales como el desgranamiento y la esperanza, que hay al respecto para resolverlo. Asimismo, pone sobre el tapete diferencias entre el rol de 'los que dicen' y de 'los que hacen' a favor del compromiso con la educación, esto es, entre quienes proponen y quienes se encuentran día a día en las aulas promoviendo experiencias excelentes. El autor nos invita a repensar el compromiso desde las aulas, atender las experiencias reales y a considerar perspectivas diversas en diferentes épocas, múltiples contextos y distintos actores que participan del hacer escolar.

El segundo capítulo, escrito por Romina Cecilia Elisondo, se titula Compromiso y creatividad en contextos educativos: relaciones necesarias. En un comienzo, la autora traza posibles relaciones entre los constructos de creatividad y compromiso académico, y plantea algunas preguntas y supuestos preliminares. Luego, y con base en una cuidadosa revisión bibliográfica realizada desde perspectivas contextuales y socioculturales, define dichos constructos, describe la relación que se da entre los mismos y brinda orientaciones generales sobre acciones e intervenciones promotoras de compromiso creativos (constructo integrador) en contextos educativos. El capítulo concluye respondiendo a las preguntas iniciales, a saber: "¿Cómo se relacionan creatividad y compromiso en contextos educativos? ¿Los entornos creativos potencian el compromiso académico de docentes y estudiantes? ¿Cómo potenciar el compromiso y la creatividad en instituciones educativas?" (Elisondo, 2020, p. 21). Se destaca un vínculo estrecho entre compromiso y creatividad, variables que se retroalimentan mutuamente y que están inmersas en interacciones complejas entre los actores y el contexto. Así, la autora focaliza la idea según la cual el hacer y las interacciones entre diferentes actores - tanto docentes como estudiantes- pueden enriquecer el contexto educativo para originar estrategias y acciones promotoras tanto de creatividad como de compromiso.

El tercer capítulo se rotula Procesos de enseñanza y de aprendizaje: el lugar del compromiso. Pasando de la agencia a la metacognición y las funciones ejecutivas, de María Laura de la Barrera. Con un amplio marco de referencia, la autora conceptualiza el compromiso académico desde una perspectiva neurocientífica. Específicamente, presenta hallazgos vinculados con el campo educativo y desmitifica algunos supuestos en los avances neurocientíficos. En este sentido cuestiona, por ejemplo, el aplicacionismo directo. Seguido de ello, profundiza en el concepto de compromiso académico y hace especial hincapié en uno de sus factores constitutivos más recientes, esto es, la agencia personal. Al respecto, plantea el vínculo directo de esta con la metacognición y las funciones ejecutivas. De modo general define la agencia como "la capacidad de actuar intencionalmente y, por lo tanto, de lograr propósitos o metas guiados por la razón" (de la Barrera, 2020, 
p. 32). Por su parte, los planteamientos de la teoría social cognitiva (Bandura, 1997) destaca que las principales propiedades de la agencia personal son la intencionalidad, la previsión, la autorreactividad y la autorreflexión.

El gran aporte que realiza la autora en el capítulo es destacar las acciones interdependientes que se necesitan para lograr una de las grandes metas actuales de la educación: promover la autonomía y el compromiso de los estudiantes a través del establecimiento de metas propias y un hacer activo en el proceso del aprendizaje. En este sentido, el rol del docente cobra valor como 'mediador' entre los aprendizajes de los estudiantes y el contexto. Finalmente, destacamos la siguiente idea que sintetiza de alguna manera la perspectiva del capítulo en su conjunto: "A desarrollar el compromiso y la agencia, se aprende. A ser metacognitivo también. El despliegue de las funciones ejecutivas, se favorece. Es un trabajo colaborativo, de todos y con todos en un contexto que lo permita" (de la Barrera, 2020, p. 35).

La segunda sección que conforma este libro se denomina Investigar el compromiso como apuesta a la trasformación educativa. En este capítulo, Daiana Yamila Rigo desarrolla específicamente el tema del trabajo de investigación titulado Rasgos contextuales y personales en la promoción del compromiso, llevado a cabo en el marco de su tesis posdoctoral en Conicet. La autora presenta su estudio de modo sintetizado y con una estructura por secciones en los apartados que integran el trabajo original: introducción, marco teórico, estudios de campo, resultados y conclusiones. La meta primordial de este trabajo se orientó a producir avances en diferentes direcciones, entre ellas el conocimiento de los procesos de aprendizaje escolar en relación con una enseñanza promotora de compromiso afectivo, cognitivo y conductual. Por otro lado, pero en estrecha vinculación, se orientó a acciones de transferencia, específicamente a procesos de indagación, observación, estudio e implementación de experiencias educativas que permitan explorar cuáles son los rasgos contextuales y los personales que facilitan y promueven el compromiso en todas sus dimensiones. El eje transversal de este trabajo fueron las interrelaciones entre los constructos en estudio, esto es, compromiso, contexto instructivo, tareas académicas, rasgos personales —de docentes y estudiantes-y rendimiento académico.

En lo que se refiere al marco teórico, la autora deja claro qué se entiende por compromiso, particularmente desde un modelo contextual, así como la caracterización de un diseño instructivo promotor del compromiso y el papel de la evaluación en dicho diseño. De manera más precisa se describe, en primer lugar, la importancia del contexto para estudiar el compromiso a partir de un enfoque sociocultural de la educación y la necesidad de una investigación situada. Luego se presentan la conceptualización del compromiso desde el modelo 
contextual. En este sentido, se conciben influencias recíprocas entre los rasgos del contexto instruccional, las relaciones sociales, los factores personales y los resultados de aprendizaje. En tercer lugar, se caracteriza un diseño instructivo que se pensó para comprometer a los estudiantes con sus aprendizajes a través de una revisión de lo que hay que enseñar, cómo aprenden los sujetos y cómo hay que enseñar para promover entornos de aprendizajes poderosos en sus posibilidades de promover un mayor compromiso de los estudiantes. Entre los factores que contribuyen en la conformación de este tipo de contextos de aprendizaje, la autora destaca las prácticas educativas originales —o de extrañamiento-y las prácticas instructivas basadas en estrategias de autorregulación. Las tareas desafiantes, justamente, dan un rol activo a los estudiantes desde el comienzo de la tarea, pues tienen que buscar información para resolverla, participar en discusiones en clase y mantener el interés durante la misma (Rigo, Elisondo y de la Barrera, 2020). Por último, se define la evaluación vista desde dos perspectivas antagónicas: como un proceso estanco — separado de la instrucción - o en espiral —integrada en el proceso de aprendizajeque caracteriza a cada perspectiva y hace foco en la evaluación formativa, situada y ligada a la vida cotidiana. Se revisa también la conceptualización del feedback formativo y evaluación por rúbrica, porque estas herramientas son capaces de promover procesos de autorregulación de los aprendizajes en los estudiantes.

Los estudios de campo están compuestos por dos etapas: en primer lugar, un diseño cuantitativo exploratorio y descriptivo sobre el contexto de la clase; en segundo lugar, una planificación e implementación de un diseño instructivo con metodología mixta. Para cada una de las etapas referidas se describen los objetivos, los aspectos metodológicos, la modalidad de recolección de datos, los procedimientos, el análisis y los resultados obtenidos. La primera etapa está orientada a la exploración y descripción del diseño y configuración de la clase para definir rasgos que contribuyen al desarrollo del compromiso. Específicamente, la investigadora examina cinco dimensiones que se ponen en juego en el aula durante los procesos de enseñanza y de aprendizaje: el compromiso - cognitivo, conductual y afectivo-, el contexto instructivo, las creencias motivacionales, el docente y la clase. La segunda etapa de investigación está orientada a planificar e implementar un diseño instructivo que, basado en un modelo contextual sobre compromiso, busca promover la implicación de los estudiantes para favorecer su rendimiento escolar. El diseño instructivo está compuesto por tres momentos: i) diseño de la tarea escolar " $i Y$ si damos la vuelta al mundo!"; ii) proceso de evaluación formativa; iii) la rúbrica como puente entre la tarea y la evaluación.

Finalmente, la segunda sección de este libro concluye con las consideraciones finales del estudio, donde se analiza y pone en discusión el valor de la transferencia 
de los principales resultados encontrados en función de los objetivos propuestos. La primera conclusión refiere, en primer lugar, al valor del contexto para llevar a cabo un estudio acerca del compromiso, contextos naturales y reales; en segundo lugar, invita a reflexionar sobre la educación y sus estudios de manera trasversal a partir del diálogo de diversas disciplinas. Una segunda conclusión afirma que el compromiso 'se contagia' en función de los rasgos que configuran a las clases. Esto pone de manifiesto la relevancia que tiene el estudio de las diferentes dimensiones de la clase para comprender la complejidad que supone planificar prácticas de enseñanza en busca de aprendizajes comprometidos. Según la autora, los estudiantes valoran las tareas que se le tornan desafiantes y con valor de utilidad, donde se promueva la autonomía a través de una estructura clara de trabajo y retroalimentaciones destinadas a mejorar los procesos de aprendizaje en un contexto de intercambios enriquecidos entre los actores.

La tercera y última conclusión sugiere que los estudiantes valoran positivamente la experiencia implementada en sus diferentes momentos. El diseño se planteó como un ciclo entrelazado interactivo entre la instrucción y la evaluación, puramente formativa, con preponderancia de la autonomía de los estudiantes y una estructura dinámica. Los estudiantes tuvieron un rol activo durante el desarrollo de la actividad, desde el momento de plantar criterios en las rúbricas para planificar, monitorear y reflexionar a lo largo del proceso, favoreciendo así la autorregulación, hasta la retroalimentación de los procesos de aprendizaje y del proceso instructivo del docente con miras a su redefinición y mejora. Todo diseño de clase es un plan de carácter sistemático que promueve el logro de metas y objetivos educativos, donde los principales componentes son los estudiantes, los recursos, los contextos y los docentes en interrelación permanente.

Esta obra, a nuestro juicio, realiza una doble contribución: i) la conceptualización enriquecida del compromiso desde diferentes perspectivas y en la implementación de diseño de instructivos que lo promuevan; y ii) la transferencia de la investigación al campo educativo, la cual resalta el valor de la investigación educativa situada con actitud crítica y promotora de cambio, revisa lo ya realizado y lo resignifica. La obra nos aporta, en su totalidad, herramientas para comprender, repensar y cuestionar los procesos de enseñanza y de aprendizaje como modo posible de avanzar hacia mejoras educativas. Mucho de lo que en ella se expone resulta novedoso e interesante a la hora de reflexionar sobre las propias prácticas de enseñanza con el fin de promover cambios que enriquezcan los contextos y, por ende, los procesos de aprendizaje y sus resultados.

En síntesis, se hace visible la importancia que toma el conocimiento sobre factores contextuales y personales para avanzar hacia contribuciones capaces 
de promover mejoras en las propuestas didácticas a favor de un mayor compromiso con los aprendizajes. De esta manera, se fundan experiencias de enseñanza y de aprendizaje de manera integral, enriquecedora y socializadora que convocan a los estudiantes a participar de manera activa como verdaderos protagonistas.

\section{Referencias}

Bandura, A. (1997). Self-efficacy: The exercise of control. W.H. Freeman and Company.

De la Barrera, M. L. (2020). Procesos de enseñanza y de aprendizaje: el lugar del compromiso. Pasando de la agencia a la metacognición y las funciones ejecutivas. En D. Y. Rigo (coord.), Compromiso y educación. Redefinición del aula como contexto de aprendizaje (pp. 29-36). Universitas. https://www. mediterranea-comunicacion.org/article/view/cmd13-compromiso-y-educacion-redefinicion-del-aula

Elisondo, R. C. (2020). Compromiso y creatividad en contextos educativos: relaciones necesarias. En D. Y. Rigo (coord.), Compromiso y educación. Redefinición del aula como contexto de aprendizaje (pp. 21-27). Universitas. https://www.mediterranea-comunicacion.org/article/view/cmd13-compromisoy-educacion-redefinicion-del-aula

Rigo, D. Y. (2020). Compromiso y educación. Redefinición del aula como contexto de aprendizaje. Universitas. https://www.mediterranea-comunicacion.org/article/view/cmd13-compromiso-yeducacion-redefinicion-del-aula

Rigo, D. Y., Elisondo, R. y de la Barrera, M. L. (2020). Commitment, Creativity and Brains: Perspectives on Gifted Education. En F. Ribeiro, T. Stoltz, A. Rocha y C. Costa-Lobo (eds.), Socio-Emotional Development and Creativity of Gifted Students (pp. 237 - 256). Universidad de Coimbra.

Rinaudo, M. C. (2014). Estudios sobre los contextos de aprendizaje. Arenas y Fronteras. In P. V. Paoloni, M. C. Rinaudo y A. González Fernández (comps.), Cuestiones en Psicología Educacional. Perspectivas teóricas, metodológicas y estudios de campo (pp. 163-205). Sociedad Latinoamericana de Comunicación Social (SLCS). http://www.cuadernosartesanos.org/2014/cde01.pdf 